\title{
The Uncertainty Relation for Joint Measurement of Position and Momentum
}

\author{
R. F. Werner
}

October 14, 2018

\begin{abstract}
We prove an uncertainty relation, which imposes a bound on any joint measurement of position and momentum. It is of the form $(\Delta P)(\Delta Q) \geq C \hbar$, where the 'uncertainties' quantify the difference between the marginals of the joint measurement and the corresponding ideal observable. Applied to an approximate position measurement followed by a momentum measurement, the uncertainties become the precision $\Delta Q$ of the position measurement, and the perturbation $\Delta P$ of the conjugate variable introduced by such a measurement. We also determine the best constant $C$, which is attained for a unique phase space covariant measurement.
\end{abstract}

Dedicated to Alexander S. Holevo on the occasion of His $60^{\text {th }}$ BIRThDAy

\section{Introduction}

Heisenberg's Uncertainty Relation $(\Delta Q)(\Delta P) \geq \hbar / 2$ is one of the most fundamental features of quantum theory, and is taught in even the most basic course on the subject. All too often, however, teachers succumb to the persistent bad habit of proving the relations as an inequality on variances for arbitrary state preparations, but then to go on to explain their 'physical meaning' in terms of a perturbation of the momentum of a particle caused by an approximate position measurement. Since the usual proof contains nothing of that sort, attentive students quickly get the impression that quantum uncertainty rubs off on their teachers as some kind of conceptual fuzziness. Our aim in this paper is to state the measurement aspect of uncertainty as rigorously as has become standard for the preparation aspect and, of course, to prove the corresponding inequality.

Both aspects of uncertainty go back all the way to Heisenberg's paper 1 in which the relations were first introduced, and it is perhaps instructive to disentangle the richness of Heisenberg's paper a little bit. He begins his discussion with the famous example of a position measurement on an electron by observation under a $\gamma$-ray microscope: the resolution $\Delta Q$ of such a device is of the order of the wavelength $\lambda$ of the photons. However, the interaction gives the electron a Compton kick, transferring an uncontrolled momentum of the order of the momentum of the photon, i.e., $\Delta P \sim 2 \pi \hbar /(\Delta Q)$. Heisenberg paraphrases this by saying that precisely at the moment of interaction, i.e., at the moment the electron's position "becomes known", the momentum "becomes unknown" in accordance with the Uncertainty Relation. He 
observes that this is related to the commutation relations, and announces that this "direct mathematical connection" will be demonstrated later in the paper. Disappointingly, this demonstration (on p. 180) turns out to be an order-of-magnitude discussion of the spread of Gaussian wave packets.

After Heisenberg the stringency of this demonstration was improved considerably, beginning with Kennard 2, and a version for general non-commuting quantities by Robertson 3 . These mathematical formulations fix the meaning of $\Delta P$ and $\Delta Q$ as the square root of variances, and replace Heisenberg's own notation " " for "of the order of magnitude of ..." by a rigorous inequality, in which even the constant $\hbar / 2$ is precisely optimal. Since the proof involves just an elementary application of the Schwarz inequality, it has become standard textbook material, and Heisenberg himself seems to have adopted it as the principal formulation of uncertainty in his later writings. The meaning of the Uncertainty Relations in this formulation is again summarized in Fig. 1] Obviously, they refer to two separate experiments, in the sense that to each single quantum particle either a position or a momentum measurement is applied. The preparation is the same in both cases, so the relations are best seen as a constraint on the possibility of preparing states with low variances.

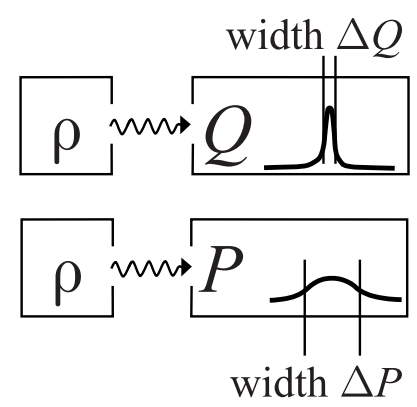

Figure 1: The Preparation Uncertainty Relation refers to the variances in two separate ideal measurements on the same state.

But what became of the microscope? Clearly, Heisenberg discusses a simple measurement process, in which the initial preparation of the electrons plays no important role. Position and momentum are both measured for the same particle (even if imperfectly). The key observation is that the measurement of position necessarily disturbs the particle, so that the momentum is changed by the measurement. Indeed, it is a fundamental theorem of quantum theory that there is no measurement without perturbation. More precisely, if the output quantum states of a measuring device coincide with the input states for all inputs, then the measured values are statistically independent of the input, i.e., no information is gained from the 'measurement'. But this statement captures none of the quantitative content of Heisenberg's discussion. Figure 2 shows how we might understand the uncertainties for the microscope: The quantum system is first subject to an approximate position measurement $Q^{\prime}$. This is not an ideal measurement $Q$, since the $\gamma$-rays have non-zero wavelength. So $\Delta Q$ is some measure of the difference between $Q$ and $Q^{\prime}$. The next step is a measurement of momentum. Due to the previous perturbation of the system, we cannot hope to recover precisely the momentum of the initial particle. So if $P^{\prime}$ is the momentum measurement (including the prior perturbation) we will see a difference $\Delta P$ to an ideal 


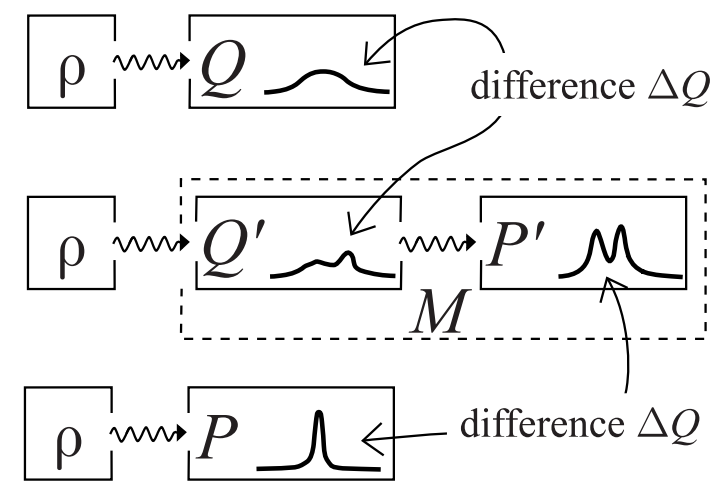

Figure 2: The Measurement Uncertainty Relations studied in this paper refers to the deviations of the marginals of a joint measurement $\mathbf{M}$ from the ideal position and momentum observables. The joint measurement can be realized by first a position measurement $\mathbf{Q}^{\prime}$ and then a momentum measurement $\mathbf{P}^{\prime}$.

momentum measurement $P$. The claim of the Measurement Uncertainty Relations is that $\Delta Q \Delta P \geq C \hbar$ for some constant $C$. The aim of this paper is to do for this relation what Kennard did for the Preparation Uncertainty Relation: to give a rigorous definition of the quantities involved and to prove the inequality as a Theorem.

The reason why this was not done 70 years ago might be that $\Delta Q$ is a difference between observables like $Q$ and $Q^{\prime}$, which are never measured in the same experiment. Therefore a quantity like the expectation of $\left(q-q^{\prime}\right)^{2}$ makes no sense at all. So we have to define $\Delta Q$ as a distance between the probability distributions of $q$ and $q^{\prime}$, which requires some conceptual work (see Section [2).

It is clear that we can make devices $M$, which for a particular input state $\rho$ produce outputs with precisely the same distributions as the ideal measurements. Indeed, we can simply make $M$ a random generator for an arbitrary pair of distributions. Such a device $M$ would utterly fail to reproduce the distributions for other input states, of course. Therefore we will define $\Delta Q$ (and similarly $\Delta P$ ) as the worst case distances between the probability distributions of $Q$ and $Q^{\prime}$.

In previous work on measurement uncertainty only covariant joint measurements were considered, i.e., measurements with the expected transformation behavior with respect to phase space translations. In this case, which will also play a crucial role in the present paper, the conceptual problem of interpreting the $\Delta \mathrm{s}$ is much easier: in that case the marginals of a joint measurement can be simulated by adding to the results of an ideal measurement some noise, which is independent of the input. Hence any parameter quantifying the size of the noise (e.g., the variance) will do. Discussions of uncertainty in this setting can be found in many places, not least in the work of Holevo [5]. The new contribution in this article is a definition of the $\Delta s$, which makes sense without covariance, and the correspondingly extended inequality.

This paper is organized as follows. We will describe the precise definitions of $\Delta P$ in Section [2] and also state our Theorem. In Section [3] we describe how to compute $\Delta P$ and $\Delta Q$ for the special case of measurements, which are covariant with respect to phase space translations, and show how to obtain the best constant $C$ in this restricted class. Finally, in Section 4 we show that general measurements $M$ never 
outperform the covariant ones, i.e., the bounds previously established also hold for joint measurements without assuming any covariance condition. Some related ideas and versions of uncertainty will be discussed in the last Section 5 of the paper.

\section{Distance of observables on a metric space}

\subsection{Monge distance of probability measures}

Let us fix some notation. If $X$ is some measurable space (i.e., a space equipped with a $\sigma$-algebra of 'measurable sets') a probability measure $\mu$ on $X$ assigns to each measurable set a probability in a countably additive way. Equivalently, we can consider the expectation value functional induced by $\mu$, i.e., $\mu(f)=\int \mu(d x) f(x)$, where $f$ is any bounded measurable function $f: X \rightarrow \mathbb{R}$. This functional, which we will denote by the same letter $\mu$ is also called the Radon measure associated with $\mu$. Whether a measure is primarily seen as a function on sets or as a linear functional is largely a matter of taste. The Radon measure point of view will have advantages in Section 4 where we need to discuss measures with non-zero weight at infinity. Hence we will use it throughout the paper. By $\delta_{x}$ we denote the point measure at $x \in X$, i.e., $\delta_{x}(f)=f(x)$ for all $f$.

A natural way of describing the difference between two probability measures $\mu_{1}$ and $\mu_{2}$ is to take the largest difference in probabilities they can assign to any event, i.e., (up to a conventional factor 2):

$$
\begin{aligned}
\left\|\mu_{1}-\mu_{2}\right\|_{1} & =2 \sup _{\sigma}\left|\mu_{1}(\sigma)-\mu_{2}(\sigma)\right| \\
& =\sup _{|f| \leq 1}\left|\mu_{1}(f)-\mu_{2}(f)\right|,
\end{aligned}
$$

where the first supremum is over all measurable sets, and the second is over all measurable functions with $|f(x)| \leq 1$ for all $x$. This quantity is known as the norm difference with respect to the norm of "total variation".

However, this distance between probability measures is totally useless for defining a quantity like $\Delta Q$. As a measurable space $X=\mathbb{R}$ and $X=\mathbb{R}^{2}$ are isomorphic, so this structure knows nothing of the topology of $X$, and of the closeness of points in $x$. For example take two point measures $\delta_{x_{1}}$ and $\delta_{x_{2}}$ for distinct points $x_{1}, x_{2} \in X$. Since there is a measurable set containing $x_{1}$ but not $x_{2}$, we always have $\left\|\delta_{x_{1}}-\delta_{x_{2}}\right\|_{1}=2$, even if the points are "very close" and so the two point measures describe practically indistinguishable probability distributions.

In order to set up a quantitative notion of the distance of probability measures, according to which nearby point measures would be close, too, we must have a notion of closeness for points to begin with. Therefore, we fix a metric $d$ on $X$. The only technical requirement linking the metric and the measurable structure is that all continuous functions for the metric are measurable. The idea is then to define the distance between probability measures as the largest difference of expectation values on "slowly varying functions".

Definition 1 Let $X$ be a metric space with metric $d$. We define the Lipshitz ball $\Lambda$ of $(X, d)$ as the set of bounded functions $f$ such that

$$
|f(x)-f(y)| \leq d(x, y)
$$


for all $x, y \in X$. Then, for any two probability measures $\mu_{1}, \mu_{2}$ on $X$ we define the distance as

$$
d\left(\mu_{1}, \mu_{2}\right)=\sup _{f \in \Lambda}\left|\mu_{1}(f)-\mu_{2}(f)\right|
$$

Strictly speaking, it is another abuse of notation to use the same letter for the metrics on points and on measures. However, the two are very closely related. For example, if we take two point measures, we find $d\left(\delta_{x}, \delta_{y}\right)=d(x, y)$, where the inequality " $\leq$ " follows by definition of the Lipshitz ball, and the reverse inequality follows by observing that the function $f(z)=d(x, z)$ (with a suitable cutoff to make it bounded) is in $\Lambda$ by the triangle inequality.

There is an alternative "dual" definition of this distance, going back to a problem by G. Monge [6] in 1781. Consider, instead of two probability distributions, two heaps of soil of equal volume and the task of transforming one heap into the other by moving around small amounts of soil. Suppose that for each such move we have to pay a price proportional to the amount and to the distance. Then the lowest possible price for the transformation is called the Monge distance between $\mu_{1}$ and $\mu_{2}$. To make this definition explicit, suppose we note for each bit of soil the initial and final location. This will result in a probability measure $\mu_{12}$ on $X \times X$, whose marginals are the given measures $\mu_{1}$ and $\mu_{2}$, respectively. The price payed will be proportional to

$$
D\left(\mu_{12}\right)=\int \mu_{12}(d x, d y) d(x, y)
$$

Clearly, for any $f \in \Lambda$, we will have

$$
\mu_{1}(f)-\mu_{2}(f)=\int \mu_{12}(d x d y)(f(x)-f(y)) \leq D\left(\mu_{12}\right) .
$$

Then the supremum of the left hand side is the distance defined in Definition [1 whereas the infimum of the right hand side is Monge distance. Due to a 1942 paper of L. Kantorovich [7] the two are, in fact, the same. This result is very much in the spirit of modern duality theory of convex optimization problems. Duality also helps to understand the structure of maximizing functions $f$ and minimizing joint distributions $\mu_{12}$, which tend to be supported on the graph of a function, provided the measures $\mu_{1}$ and $\mu_{2}$ are not too lumpy. Uniqueness can be enhanced 8 by replacing $d(x, y)$ in the objective functional by $d(x, y)^{1+\epsilon}$, thereby putting an extra penalty on scattering mass, and then letting $\epsilon \rightarrow 0$.

Position and momentum take their values in a vector space, so we will briefly note some special properties of the Monge metric in this case. We assume that the metric $d$ is consistent with the linear structure, namely translationally invariant, and homogeneous with respect to scaling. In other words, we require $d(x, y)=|x-y|$ for some vector space norm $|\cdot|$. The scaling property is important so that we can assign to distances the same physical units as to the coordinates. Another key operation that requires the vector space structure is adding noise from an independent source. On the level of probability measures this is represented by the convolution $\mu * \nu$ :

$$
(\mu * \nu)(f)=\int \mu(d x) \int \nu(d y) f(x+y) .
$$

Basic properties of the metric on probability measures are summarized in the following Lemma.

Lemma 1 Consider $X=\mathbb{R}^{n}$, with a metric d given by a vector space norm. 
1. Then for any probability measures $\mu, \nu$, we have the inequality $d(\mu, \mu * \nu) \leq \int \nu(d y)|y|$.

2. Let $n=1$, and let $F_{i}(t)=\mu_{i}((-\infty, t])$ denote the distribution functions of two probability measures on $\mathbb{R}$. Then

$d\left(\mu_{1}, \mu_{2}\right)=\int_{-\infty}^{\infty} d x\left|F_{1}(x)-F_{2}(x)\right|$.

The first estimate follows by inserting for $\mu_{12}$ in (5) the joint distribution of $x$ and $x+y$ implied by the independence of $x$ and $y$ according to (6). For the second statement note that we can take the supremum over $f \in \Lambda$ over the subset of piecewise differentiable functions with $\left|f^{\prime}(x)\right| \leq 1$ such that $f^{\prime}$ has compact support, and write

$$
\mu_{i}(f)=-\int_{-\infty}^{\infty} d x F_{i}(x) f^{\prime}(x)
$$

up to boundary terms which cancel in the difference $\mu_{1}(f)-\mu_{2}(f)$. This also provides a formula for the maximizing $f$ : we take $f^{\prime}(x)= \pm 1$, depending on the sign of $F_{1}(x)-$ $F_{2}(x)$.

\subsection{Distance of Observables}

Let us now consider observables over $X$, i.e. quantum devices, which produce an output $x \in X$ in every single experiment. Let us take the quantum particles to be described in a Hilbert space $\mathcal{H}$, so that every preparation of quantum particles is described by a density operator $\rho$. For any such preparation $\rho$, the outputs of the device are then distributed with respect to a probability measure $\mu_{\rho}$ on $X$. Since the map $\rho \mapsto \mu_{\rho}(f)$ is affine in $\rho$ and bounded, for every bounded measurable function $f$, there is an operator $\mathbf{F}(f) \in \mathcal{B}(\mathcal{H})$ such that

$$
\mu_{\rho}(f)=\operatorname{tr}(\rho \mathbf{F}(f))
$$

Then $\mathbf{F}$ is a linear operator, taking positive functions to positive operators, and $\mathbf{F}(1)=$ $\mathbb{1}$. Evaluated just on the indicator functions we get a positive operator values measure (POVM), from which the values for general $f$ are recovered by integration: We have $\mathbf{F}(f)=\int \mathbf{F}(d x) f(x)$. Either the measure or the linear operator $\mathbf{F}$ will called an observable, and the two are denoted by the same letter. Of course, an important special case is that each value of the measure is a projection, which is equivalent to $\mathbf{F}(f g)=\mathbf{F}(f) \mathbf{F}(g)$. Such observables will be called projection valued (PVM).

How should we define the distance of observables now? An approach based on joint distributions is not feasible, because very often positive operator valued measures do not admit an extension to a joint observable, so the measured outputs of two observables typically cannot be seen in the same experiment and compared for each single shot separately. We can, however, compare distributions. And since equality of observables $\mathbf{F}_{1}, \mathbf{F}_{2}$ means, by definition, that the probability measures $\mu_{1, \rho}$ and $\mu_{2, \rho}$ coincide for every $\rho$, it is natural to say that two observables are similar if they give similar probability distributions for all states, in the sense of the metric defined previously. Hence we set, for any two observables $\mathbf{F}_{1}, \mathbf{F}_{2}$ on the same metric output space $(X, d)$ and for quantum systems with the same Hilbert space $\mathcal{H}$ :

$$
\begin{aligned}
d\left(\mathbf{F}_{1}, \mathbf{F}_{2}\right) & =\sup _{\rho} \sup _{f \in \Lambda} \mid \operatorname{tr}\left(\rho\left(\mathbf{F}_{1}(f)-\mathbf{F}_{2}(f)\right) \mid\right. \\
& =\sup _{f \in \Lambda}\left\|\mathbf{F}_{1}(f)-\mathbf{F}_{2}(f)\right\|
\end{aligned}
$$


where the supremum over $\rho$ in the first line is over all density operators, and in the second line we used that, for hermitian operators $A$ we can express the operator norm as $\|A\|=\sup _{\rho}|\operatorname{tr}(\rho A)|$. Thus $" d\left(\mathbf{F}_{1}, \mathbf{F}_{2}\right) \leq \varepsilon "$ is synonymous with the bound $\left|\operatorname{tr}\left(\rho \mathbf{F}_{1}(f)\right)-\operatorname{tr}\left(\rho \mathbf{F}_{2}(f)\right)\right| \leq \varepsilon$, on differences of expectation values, valid for all states $\rho$, and all bounded functions $f$ with Lipshitz slope at most 1 .

It is important to note that the dual characterization of the metric as Monge distance cannot be transferred from the case of scalar probability measures to the operator valued case. Of course, for any fixed $\rho$ we get a joint probability distribution $\mu_{12, \rho}$ minimizing cost for the Monge problem of $\mu_{1, \rho}$ and $\mu_{1, \rho}$. However, in contrast to the $\mu_{i, \rho}$, the function $\rho \mapsto \mu_{12, \rho}$ is not affine in $\rho$, and hence there is no observable $\mathbf{F}_{12}$ such that $\mu_{12, \rho}(f)=\operatorname{tr}\left(\rho \mathbf{F}_{12}(f)\right)$, and even if there happens to be some joint measurement $\mathbf{F}_{12}$ with marginals $\mathbf{F}_{i}$, providing an affine family of joint distributions, this gives only a loose upper bound $d\left(\mathbf{F}_{1}, \mathbf{F}_{2}\right) \leq\left\|\mathbf{F}_{12}(d)\right\|$.

\subsection{Statement of the Theorem}

Let us now consider a quantum mechanical system with $n$ canonical degrees of freedom, described in a Hilbert space $\mathcal{H}$. That is there are self adjoint operators $P_{\mu}, Q_{\mu}, \mu=$ $1, \ldots, n$ satisfying the canonical commutation relations

$$
\begin{aligned}
i\left[P_{\mu}, Q_{\nu}\right] & =\hbar \delta_{\mu \nu} \mathbb{I} \\
i\left[P_{\mu}, P_{\nu}\right] & =i\left[Q_{\mu}, Q_{\nu}\right]=0
\end{aligned}
$$

on a dense set of vectors, on which all real linear combinations of these operators are essentially self-adjoint. Note that we do admit additional degrees of freedom unrelated to the $P_{\mu}, Q_{\mu}$ under consideration. Under these conditions there are joint spectral measures for the $Q_{\mu}$, i.e., there is a unique projection valued observable on $X=\mathbb{R}^{f}$ such that $Q_{\mu}=\int \mathbf{Q}(d x) x_{\mu}$. As a metric on $X$ we take some metric derived from a norm $|\cdot|$ on position space, such as the Euclidean metric when we consider a single particle. By $|Q|$ we denote the operator

$$
|Q|=\mathbf{Q}(|\cdot|)=\int \mathbf{Q}(d x)|x| .
$$

Similarly, we consider the momentum observable $\mathbf{P}$, which is the joint spectral measure of the $P_{\mu}$, and choose a suitable norm on momentum space.

Theorem. Let $\mathbf{Q}, \mathbf{P}$ be the position and momentum observables of a system with $n$ degrees of freedom in a Hilbert space $\mathcal{H}$. Let $\mathbf{M}$ be an observable on $\mathbb{R}^{n} \times \mathbb{R}^{n}$ with marginals $\mathbf{M}_{1}$ and $\mathbf{M}_{2}$. Then

$$
d\left(\mathbf{Q}, \mathbf{M}_{1}\right) \cdot d\left(\mathbf{P}, \mathbf{M}_{2}\right) \geq C \hbar .
$$

The best constant $C$ in this inequality is determined as $C \hbar=E_{0}^{2} / 4 a b$, where $E_{0}$ is the lowest eigenvalue of the operator

$$
K=a|Q|+b|P|
$$

for some positive constants $a, b>0$. Equality in (12) holds for a suitable covariant observable. 
Of course, the numerical value of the constant $C$ does depend on the two metrics chosen, and on the number $n$ of degrees of freedom. For a single degree of freedom, with $|\cdot|$ the usual absolute value we get

$$
C \approx 0.304745
$$

The unique covariant observable attaining this bound is determined (numerically) in Section 3.2 It is not equal to the covariant observable based on coherent states, which realizes the uncertainty product $C^{\prime}=1 / \pi \approx 0.3183$.

\subsection{Weight at infinity}

Before going into the proof of the Theorem, we have to be a bit more precise about the class of functions $f$, for which we need expectations $\mu(f)$ and operators $\mathbf{F}(f)$ to be defined. This subsection is somewhat technical, and can be skipped by those who are only interested in the construction of joint measurements saturating the bound.

The issues we discuss in this subsection do not require covariance, and make sense for a general locally compact metric space $(X, d)$, in our case either phase space, position space or momentum space. Integration on locally compact spaces can be developed nicely as a theory of linear functionals ("Radon measures") on the space $\mathcal{C}_{00}(X)$ of continuous functions of compact support. This approach is advocated e.g., by Bourbaki [9] (see also Dieudoné[10] for the simpler special case of metrizable separable spaces, which is all we need here).

However, we need expectation values not just for $f \in \mathcal{C}_{00}(X)$. For example, in order to define the normalization of probability measures we need to integrate the function " 1 ". A large part of measure and integration theory is devoted to extending the definition of integrals to larger and larger classes of functions. For the normalization one defines

$$
\mu(1)=\sup \left\{\mu(f) \mid f \in \mathcal{C}_{00}(X), \quad f \leq 1\right\} .
$$

The extension of $\mu(f)$ to all bounded measurable functions $f$ follows similar limit processes. For our purposes, however, it is only necessary to evaluate expectations on $f \in \Lambda$, so we can compute the distance of probability measures via Definition [1] Therefore we will stay with the minimal space of functions necessary for that purpose, which is the $\mathrm{C}^{*}$-algebra generated by $\Lambda$, the algebra $\mathcal{C}_{\mathrm{uc}}(X)$ of bounded uniformly continuous functions on $X$.

Now in Section 4 we will construct directly some normalized positive linear functionals (i.e., "states") $\mu$ on $\mathcal{C}_{\mathrm{uc}}(X)$, and we would like to conclude that such a $\mu$ defines a measure on $X$. The problem is, however, that since $1 \in \mathcal{C}_{\mathrm{uc}}(X)$, an equation like (15) is now no longer a definition of the left hand side, but a property of the functional $\mu$. And we will see that it may indeed fail to be true. In other words, for such functionals the monotone convergence theorem $\sup _{n} \mu\left(f_{n}\right)=\mu\left(\sup _{n} f_{n}\right)$ for the pointwise supremum of functions on $X$ may fail.

How does this fit in with the equivalence between 'measures as set functions' and 'measures as linear functionals' proclaimed at the beginning of this subsection and in Section 22. This can be understood by considering the example of the algebra $\mathcal{A}$ of bounded continuous functions on the open unit disk, which have a continuous extension to the closed disk. As an algebra this is identical with the continuous functions on the closed disk, but what is meant by 'pointwise supremum' now depends on what we consider as the domain of these functions. For example, a point measure on the boundary has the property of giving zero expectation to any function with compact 
support inside the open disc, producing a failure Eq. (15). It is clear from this example that the equivalence between measures as set functions and as linear functionals may require that we suitably extend the underlying space, i.e., we consider measures on the closed disc rather than just the open disc.

The general situation for a locally compact metric space $X$ is quite similar to this example. Like every commutative $\mathrm{C}^{*}$-algebra, the algebra $\mathcal{C}_{\mathrm{uc}}(X)$ is isomorphic to the continuous functions $\mathcal{C}(\widehat{X})$ on a compact space $\widehat{X}$, called the Gelfand spectrum of $\mathcal{C}_{\mathrm{uc}}(X) . \quad \widehat{X}$ can be constructed as the set of pure states on the algebra $\mathcal{C}_{\mathrm{uc}}(X)$. Since evaluation at a phase space point is a pure state, we have $X \subset \widehat{X}$, and $\widehat{X}$ is a compactification of $X$. The additional points $\widehat{X} \backslash X$ should be thought of as points at infinity, and clearly a measure may be supported on such points so that the restriction of $\mu$ to $\mathcal{C}_{00}(X)$ is zero, and Equation (15) is violated as $1 \neq 0$. The points at infinity have a very rich structure 11, but in this paper we are only interested in their collective weight with respect to a probability measure, which is simply the difference between left and right hand side of Equation (15): For the overall weight at infinity of a Radon probability measure $\mu$ on $\mathcal{C}_{\mathrm{uc}}(X)$ we introduce the notation

$$
\mu(\infty)=1-\sup \left\{\mu(f) \mid f \in \mathcal{C}_{00}(X), \quad f \leq 1\right\}
$$

For a positive operator valued measure we can take exactly the same definition: the supremum exists in the weak operator topology, because the net of functions $f$ is directed. Equivalently, we can apply the scalar definition to every measure $\mu_{\rho}(f)=$ $\operatorname{tr}(\rho \mathbf{M}(f))$, and define the operator weight at infinity by $\operatorname{tr}(\rho \mathbf{M}(\infty))=\mu_{\rho}(\infty)$ for every $\rho$. The key observation, allowing us later to eliminate weights at infinity, is the following

\section{Lemma 2}

1. For any Radon probability measures $\mu_{1}, \mu_{2}$ on a locally compact metric space $(X, d): \quad d\left(\mu_{1}, \mu_{2}\right)<\infty$ implies $\mu_{1}(\infty)=\mu_{2}(\infty)$.

2. Let $\mathbf{M}$ be an observable on phase space, whose marginals have finite distance to the standard position and momentum observables, respectively. Then $\mathbf{M}(\infty)=$ 0 .

Proof: $\quad$ As a a net of functions $f_{R} \in \mathcal{C}_{00}(X)$ we choose

$$
f_{R}(x)=\left\{\begin{array}{cll}
1-d(0, x) / R & \text { if } & d(0, x) \leq R \\
0 & \text { if } & d(0, x)>R
\end{array}\right.
$$

where $0 \in X$ is an arbitrarily chosen reference point. Since a locally compact metric space is the union of the compact balls $\{x \mid d(0, x) \leq R\}$ this family eventually dominates every function $f \in \mathcal{C}_{00}(X)$ with $f \leq(1-\varepsilon)$. Hence $1-\mu(\infty)=\lim _{R \rightarrow \infty} \mu\left(f_{R}\right)$ for every probability measure. Then, since $R f_{R} \in \Lambda$,

$$
\left|\mu_{1}\left(f_{R}\right)-\mu_{2}\left(f_{R}\right)\right| \leq \frac{1}{R} d\left(\mu_{1}, \mu_{2}\right)
$$

and the first result follows by taking the limit $R \rightarrow \infty$.

For the second statement consider the inequality

$$
\begin{aligned}
1-f_{R}(p) f_{R}(q) & =\left(1-f_{R}(p)\right)+f_{R}(p)\left(1-f_{R}(q)\right) \\
\leq & \left(1-f_{R}(p)\right)+\left(1-f_{R}(q)\right),
\end{aligned}
$$


apply $\mathbf{M}$ and take the limit $R \rightarrow \infty$ to get $\mathbf{M}(\infty) \leq \mathbf{M}_{1}(\infty)+\mathbf{M}_{2}(\infty)$, where $\mathbf{M}_{i}$ are the two marginals. But since the standard position and momentum observables have zero weight at infinity, part 1 of the Lemma shows that $\mathbf{M}_{i}(\infty)=0$.

\section{Covariant observables}

\subsection{Phase space covariant observables}

It is not a priori clear that there exist approximate joint measurements of $P$ and $Q$, making $\Delta Q=d\left(\mathbf{Q}, \mathbf{M}_{1}\right)$ and $\Delta P=d\left(\mathbf{P}, \mathbf{M}_{2}\right)$ finite. But there is a simple, and even well-known construction for joint measurements of position and momentum achieving just that. These phase space observables have the additional property, that the unitary groups of translation (generated by the momentum operators) and boosts (generated by the position operators) act like a shift in phase space on the arguments of $\mathbf{M}$. Let us introduce the Weyl operators (phase space translations)

$$
W(p, q)=\exp \frac{i}{\hbar}(q \cdot P-p \cdot Q)
$$

We will assume in this section that beyond the canonical ones under consideration there are no additional degrees of freedom, i.e., the Weyl operators act irreducibly on $\mathcal{H}$. Then by von Neumann's Uniqueness Theorem[12] we can take, up to unitary equivalence, $\mathcal{H}=\mathcal{L}^{2}\left(\mathbb{R}^{n}, d x\right), Q_{\mu}$ the multiplication by the coordinate $x_{\mu}$ and $P_{\mu}=$ $\frac{\hbar}{i} \frac{\partial}{\partial x_{\mu}}$. The Weyl operators in this representation become

$$
(W(p, q) \psi)(x)=\exp \frac{i}{\hbar}\left(-\frac{p \cdot q}{2}-p \cdot x\right) \psi(x+q) .
$$

Then, denoting by $\left(\tau_{x} f\right)(y)=f(y-x)$ the translate of a function on a vector space by $x$, we get the shift covariance property of the standard position observable becomes

$$
\mathbf{Q}\left(\tau_{q} f\right)=W(p, q)^{*} \mathbf{Q}(f) W(p, q),
$$

for all bounded measurable $f$ and all $p, q \in \mathbb{R}^{n}$. There is an analogous property of $\mathbf{P}$, and we define a covariant phase space observable by the equation

$$
\mathbf{M}\left(\tau_{(p, q)} f\right)=W(p, q)^{*} \mathbf{M}(f) W(p, q) .
$$

It turns out that there is a closed formula for all such observables, described in the following Lemma. Recall from the previous section that $\mathcal{C}_{\mathrm{uc}}(X)$ denotes the algebra of bounded uniformly continuous functions on phase space $X$, and $\mathcal{C}_{00}(X)$ the subalgebra of functions with compact support. The Lemma is well known, and versions of it can be found in ,PHyUni[13, 14 15].

Lemma 3 Let $M$ be a covariant observable on phase space, i.e., a linear map $\mathbf{M}$ : $\mathcal{C}_{\mathrm{uc}}(X) \rightarrow \mathcal{B}(\mathcal{H})$, taking positive functions to positive operators, and satisfying Eq. (22). Suppose that $\mathbf{M}$ has zero weight at infinity, i.e., $\sup \left\{M(f) \mid f \in \mathcal{C}_{00}(X), f \leq 1\right\}=\mathbb{I}$. Then there is a positive operator $\mathbf{m}$ with $\operatorname{tr}(\mathbf{m})=1$ such that

$$
\mathbf{M}(f)=\int \frac{d p d q}{(2 \pi \hbar)^{n}} f(p, q) W(p, q)^{*} \mathbf{m} W(p, q) .
$$


Conversely, this formula defines a covariant observable for every $\mathbf{m}$. The integral is a weak integral, i.e., for every density operator $\rho$ we have to compute the expectation as

$$
\operatorname{tr}(\rho \mathbf{M}(f))=\int \frac{d p d q}{(2 \pi \hbar)^{n}} f(p, q) \operatorname{tr}\left(\rho W(p, q)^{*} \mathbf{m} W(p, q)\right) .
$$

Here the operator $\mathbf{m}$ is appropriately called a density operator for two separate reasons: on the one hand as positive operator of trace 1, and on the other hand as the "Radon-Nikodym derivative" of the observable at the origin. The fact that the trace under the integral is an integrable function, and, in fact a probability density on phase space follows from the fundamental "square integrability" property of Weyl operators. In the special case that $\mathbf{m}$ is a coherent state (ground state of an oscillator Hamiltonian) this probability density is also known as the Husimi function 4 of $\rho$.

It is now easy to compute the marginals of $\mathbf{M}$. Of course, the marginals $\mathbf{M}$ will inherit a covariance property: For example, the position-like marginal $\mathbf{M}_{1}$ will have the same property (21) as the position observable. Since each $\mathbf{M}_{1}(f)$ commutes with momentum translations, these operators must be functions of position, and the covariance for position shifts forces $\mathbf{M}_{1}$ to be equal to $\mathbf{Q}$ up to some smearing by convolution with a fixed probability density. Explicitly, we get the required density from the form of the Weyl operators. The result is

$$
m^{Q}(f)=\operatorname{tr}\left(\Pi^{*} \mathbf{m} \Pi \mathbf{Q}(f)\right),
$$

where $\Pi$ is the parity operator $(\Pi \psi)(x)=\psi(-x)$. With the analogous expression for momentum and the definition (6) of convolution we then have

$$
\mathbf{M}_{1}=\mathbf{Q} * m^{Q} \quad \text { and } \quad \mathbf{M}_{2}=\mathbf{P} * m^{P} .
$$

To summarize: the marginals of a covariant phase space observable can be simulated in the following way: one simply makes the corresponding ideal position or momentum measurement, and adds some noise from a source which independent of the quantum state. The noise distributions $m^{Q}$ and $m^{P}$ are the position and momentum distributions of a density operator (namely $\Pi^{*} \mathbf{m} \Pi$ ), hence there is the usual tradeoff: if we insist on a good position measurement, i.e., sharply peaked $m^{Q}$, then $m^{P}$ will be very spread out, and much noise is added to momentum, and conversely. In the following section we make this quantitative in the sense of the distance of observables.

\subsection{Optimizing over covariant observables}

The uncertainties $d\left(\mathbf{M}_{1}, \mathbf{Q}\right)$ follow from Eq. (26) and Lemma 1 we get

$$
d\left(\mathbf{M}_{1}, \mathbf{Q}\right)=\int m^{Q}(d x)|x|=\operatorname{tr}(\mathbf{m}|\mathbf{Q}|),
$$

and the analogous relation for $P$. Here the inequality " $\leq$ " follows from Lemma 11 , and equality follows from the formula $d\left(\delta_{y}, \nu\right)=\int \nu(d x) d(x, y)$ and the observation that there are states $\rho$ whose position distribution is arbitrarily close to a point measure. We now have to determine which combinations of two positive numbers

$$
\left(d\left(\mathbf{M}_{1}, \mathbf{Q}\right), d\left(\mathbf{M}_{2}, \mathbf{P}\right)\right)=(\operatorname{tr}(\mathbf{m}|\mathbf{Q}|), \operatorname{tr}(\mathbf{m}|\mathbf{P}|))
$$


can be obtained by varying the density operator $\mathbf{m}$. Because the coordinates depend linearly on $\mathbf{m}$, this is a convex set in the plane. Moreover, if one pair $\left(\delta_{1}, \delta_{2}\right)$ is possible, then so is $\left(\delta_{1}^{\prime}, \delta_{2}^{\prime}\right)$ with $\delta_{i}^{\prime} \geq \delta_{i}$, because we can replace $\mathbf{m}$ by a suitable average over translates, and can vary the distribution of translation vectors from sharply concentrated to very broad. An important point is that we can also apply the dilation symmetry $Q_{\nu} \mapsto \lambda Q_{\nu}, P_{\nu} \mapsto \lambda^{-1} P_{\nu}$. This is shown in Figure 3.2 with every point the admissible region contains the entire hyperbola through that point. In order to

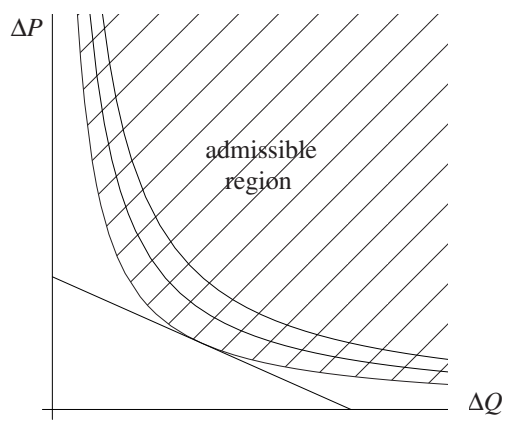

Figure 3: Admissible region for pairs $(\Delta Q, \Delta P)=(\operatorname{tr}(\rho|\mathbf{Q}|), \operatorname{tr}(\rho|\mathbf{P}|))$. The tangent shown is the contour line of $a \Delta Q+b \Delta P$ realizing the minimum expectation of $K$.

find the parameter $C \hbar$ for the boundary hyperbola, consider the lowest admissible expectation $E_{0}$ for a linear combination

$$
K=a|\mathbf{Q}|+b|\mathbf{P}|,
$$

with $a, b>0$. This is the same as the smallest $a \Delta Q+b \Delta P$ with $(\Delta Q, \Delta P)$ in the admissible region of Figure 3.2 Clearly this will be attained on the boundary hyperbola $(\Delta Q)(\Delta P)=C \hbar$, which gives $E_{0}=2 \sqrt{a b C \hbar}$. Solving for $C$ we find the statement of the Theorem, for the special case of covariant $\mathbf{M}$.

We still have to clarify whether the bound is attained, i.e., whether there is really an eigenvalue $E_{0}$ at the bottom of the spectrum of $K$. This is equivalent to the same question for the top of the spectrum of the operator $(K+\mathbb{I})^{-1}$, and we will show it by verifying that $(K+\mathbb{I})^{-1}$ is a compact operator. This also shows that the relevant eigenvalue has finite multiplicity. A quick way to show compactness is by the correspondence theory of Ref 15, 11]: we only need to show that $(p, q) \mapsto$ $W(p, q)^{*}(K+\mathbb{I})^{-1} W(p, q)$ is continuous in norm (which is obvious by a resolvent equation), and that the function

$$
k(p, q)=\left\langle W(p, q) \Phi,(K+\mathbb{I})^{-1} W(p, q) \phi\right\rangle
$$

goes to zero at infinity, when $\Phi$ is some fixed Gaussian wave function. But since the operator inverse is decreasing with respect to operator ordering, we have $(K+\mathbb{I})^{-1} \leq$ $(a|P|+\mathbb{I})^{-1}$, from which we get the estimate $k(p, q) \leq \operatorname{const}(a|p|+1)^{-1}$, and similar estimate for $q$. Hence $k$ goes to zero.

In practice the computation of $E_{0}$ is best done by using the symmetry of the problem. For several degrees of freedom and Euclidean norms this is rotation symmetry, 
for one degree of freedom, we still have reflection symmetry. In addition, it is useful to take $a=b=\hbar=1$, and use the Fourier transform symmetry of $K$. Since the $E_{0}$-eigenspace is finite dimensional, we can seek joint eigenvectors of $K$ and the symmetries. Then $K$ is truncated to a subspace spanned by finitely many eigenfunctions of the harmonic oscillator with fixed symmetry, and the resulting matrix (which can be constructed symbolically, i.e., with infinite precision) is numerically diagonalized. One readily finds that the ground state of $K$ is close to the oscillator ground state (see Fig. 3.2), and is realized for angular momentum $\ell=0$ (resp. even parity), and vectors invariant under Fourier transform. For more than one degree of freedom we get the

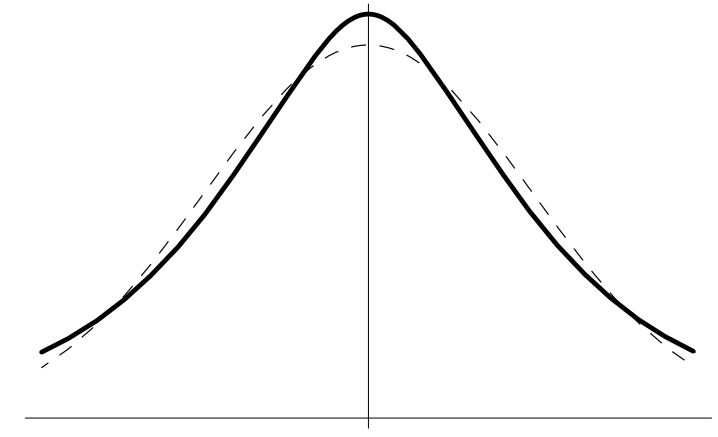

Figure 4: The ground state wave function of the operator $K$ (solid line), compared to the Gaussian oscillator ground state (dashed) for one degree of freedom.

following table

\begin{tabular}{c|c|r} 
dimension & $C$ & $C^{\prime}$ \\
\hline 1 & 0.3047 & $1 / \pi=0.3183$ \\
2 & 0.7628 & $\pi / 4=0.7853$ \\
3 & 1.2457 & $4 / \pi=1.2732$ \\
42 & 20.710 & 20.751
\end{tabular}

\section{Reduction to the covariant case}

In this section we will prove that in order to construct a joint measurement $\mathbf{M}$ with small uncertainties we can restrict attention to the covariant observables studied in the previous section. The basic idea is to average over phase space translations, thus turning a given observable $\mathbf{M}$ into a covariant one $\mathbf{M}^{\text {av }}$ with at least as small error bounds.

The basis for the construction is a so-called invariant mean [16] on the group of phase space translations: this associates to any bounded continuous function $f$ on phase space a number $\eta(u)$ such that $u \mapsto \eta(u)$ is linear, positive on positive functions, normalized as $\eta(1)=1$, and invariant in the sense that $\eta\left(\tau_{x} u\right)=\eta(u)$. The existence of invariant means is by no means obvious. Any constructive procedure based on integrating over larger and larger sets, and dividing by the volume of the set, will be convergent only for 'well behaved' functions, such as almost periodic ones or functions going to zero at infinity. The latter always average to zero, i.e., as a set an invariant mean has weight at infinity $\eta(\infty)=1$ in the sense of Eq. (16). A functional $\eta$ defined 
on all bounded continuous functions can indeed not be constructed explicitly, and we know its existence only via the axiom of choice.

Now let $f$ be a uniformly continuous function on phase space, and $\rho$ a density operator. Then we define the operator $\mathbf{M}^{\text {av }}(f)$ by

$$
\begin{aligned}
\operatorname{tr}\left(\rho \mathbf{M}^{\mathrm{av}}(f)\right) & =\eta(u(\rho, f)), \\
\text { where } u(\rho, f)(p, q) & =\operatorname{tr}\left(W(p, q)^{*} \rho W(p, q) \mathbf{M}\left(\tau_{(p, q)} f\right)\right)
\end{aligned}
$$

The function $u(\rho, f)$ is designed so that it is constant, if $\mathbf{M}$ is covariant, in which case case $\mathbf{M}^{\text {av }}=\mathbf{M}$. For arbitrary $\mathbf{M}$ it is still always uniformly continuous, because for uniformly continuous functions $f$ translation is norm continuous, and because Weyl operators are strongly continuous in $(p, q)$, making $(p, q) \mapsto W(p, q) \rho W(p, q)^{*}$ continuous in trace norm. The function $u$ is also bounded by the upper bound $\|f\|_{\infty}$ for $f$. It follows that the invariant mean $\eta$ is applicable, and that the right hand side (30) is a bounded linear functional on the convex set of density operators, and, consequently, there is a unique bounded operator $\mathbf{M}^{\text {av }}(f)$. Obviously, $\mathbf{M}^{\text {av }}(f)$ is linear in $f$, positive on positive functions, and normalized $\left(\mathbf{M}^{\mathrm{av}}(1)=\mathbb{I}\right)$. By invariance of the mean it is also evident that it is a covariant observable.

The crucial point we have to establish now is that averaging does not increase uncertainty. To this end, let us consider the set $\mathcal{M}\left(\delta_{1}, \delta_{2}\right)$ of observables $\mathbf{M}$ with $d\left(\mathbf{Q}, \mathbf{M}_{1}\right) \leq \delta_{1}$ and $d\left(\mathbf{P}, \mathbf{M}_{2}\right) \leq \delta_{2}$. In other words, we take $\mathcal{M}\left(\delta_{1}, \delta_{2}\right)$ as the set of observables $\mathbf{M}$ on phase space, such that for all density operators $\rho_{1}, \rho_{2}$ and all Lipshitz functions $f_{1}, g_{1}: \mathbb{R}^{n} \rightarrow \mathbb{R}$ with $f, g \in \Lambda$ :

$$
\begin{aligned}
& \operatorname{tr}\left(\rho_{1} \mathbf{M}_{1}\left(f_{1}\right)\right)-\operatorname{tr}\left(\rho_{1} \mathbf{Q}\left(f_{1}\right)\right) \leq \delta_{1} \\
& \operatorname{tr}\left(\rho_{2} \mathbf{M}_{2}\left(f_{2}\right)\right)-\operatorname{tr}\left(\rho_{2} \mathbf{P}\left(f_{2}\right)\right) \leq \delta_{2} .
\end{aligned}
$$

Suppose an observable $\mathbf{M}$ satisfies these bounds. Then these relations remain true, if we replace $\rho$ by $W(p, q) \rho W(p, q)^{*}$, and the functions $f, g$ by appropriately shifted ones. The terms involving $\mathbf{P}$ and $\mathbf{Q}$ are unchanged by this, but the terms with $\mathbf{M}$ become continuous functions of $(p, q)$ of the kind $u(\rho, f)$ in (30), to which we may apply the invariant mean $\eta$. As a result we find that

$$
\mathbf{M} \in \mathcal{M}\left(\delta_{1}, \delta_{2}\right) \Rightarrow \mathbf{M}^{\mathrm{av}} \in \mathcal{M}\left(\delta_{1}, \delta_{2}\right) .
$$

Hence without increasing the uncertainty bounds we may replace $\mathbf{M}$ by the covariant observable $\mathbf{M}^{\mathrm{av}}$.

This reduces the problem of characterizing the admissible pairs $\left(\delta_{1}, \delta_{2}\right)$ to Section 3 except for two issues. The first is that throughout that section we had assumed the Weyl operators to act irreducibly, i.e., there were no further degrees of freedom present. However, von Neumann's uniqueness Theorem[12] asserts that any system of Weyl operators can be decomposed into a direct sum of irreducible systems. Let $p_{\alpha}$ be the projections onto the irreducible direct summands, which by definition commute with all Weyl operators. Then we claim that $\mathbf{M} \in \mathcal{M}\left(\delta_{1}, \delta_{2}\right)$ implies that $\sum_{\alpha} p_{\alpha} \mathbf{M} p_{\alpha} \in$ $\mathcal{M}\left(\delta_{1}, \delta_{2}\right)$. The argument for this is averaging as before, but over the group of unitaries of the form $U=\sum_{\alpha} u_{\alpha} p_{\alpha}$ with $\left|u_{\alpha}\right|=1$. Clearly, such a direct sum of observables lies in $\mathcal{M}\left(\delta_{1}, \delta_{2}\right)$ iff every summand does. On the one hand, this means that additional degrees of freedom cannot increase the set of admissible $\left(\delta_{1}, \delta_{2}\right)$, and on the other hand it means that if such a pair is admissible for irreducible Weyl systems, we can construct observables with this bound for arbitrary systems as direct sums. 
The second issue we have to address is that $\mathbf{M}^{\text {av }}$ comes out as a Radon observable, i.e., we get an operator $\mathbf{M}^{\text {av }}(f)$ for every bounded uniformly continuous function $f$, but such an observable might have non-zero weight at infinity. In fact, it is typical for constructions based on a compactness argument (such as our appeal to the existence of invariant means) that one has to verify in the end that the construction does not lead to a wild element of a compactified space. For example, if we had omitted the Weyl operators from the definition of $u$, we could have still obtained some observable $\mathbf{M}^{\text {av }}$ from Eq. (30) by averaging. But rather than getting a covariant observable we would have found the observable $\widetilde{\mathbf{M}^{\mathrm{av}}}(f)=\eta(f) \mathbb{I}$ which has only weight at infinity. This is the reason we had to discuss weights at infinity in Section 2.4 In fact all the hard work was already done there: When $\delta_{1}$ and $\delta_{2}$ are finite, $\mathbf{M}^{\text {av }} \in \mathcal{M}\left(\delta_{1}, \delta_{2}\right)$, implies that $\mathbf{M}^{\mathrm{av}}(\infty)=0$ by Lemma 2]2, and therefore, by Lemma 31 that we can construct $\mathbf{M}^{\mathrm{av}}$ by integration over a density operator, and compute and optimize the uncertainties as in Section 3.2 This concludes the proof of the Theorem.

\section{$5 \quad$ Other Uncertainty Relations}

Of course, all uncertainty relations are related. Some variants that are closely related to the present paper will be briefly commented in this section.

\subsection{Measurement as Preparation}

One way of reducing measurement uncertainty to preparation uncertainty is the projection postulate: According to this postulate the state of a system after measurement is an eigenstate of the measured observable for that eigenvalue, which happened to be the outcome of the measurement. Let us assume some approximate version of this postulate holds for the approximate measurement $Q^{\prime}$ for the microscope (Of course, this restricts the applicability of our argument). Then by conditioning on the particular value of $q^{\prime}$ obtained from the $Q^{\prime}$-measurement, we could understand the position measurement as a preparation of states with position and momentum spreads $\approx \Delta Q$ and $\Delta P$. The relation between $\Delta Q$ and $\Delta P$ would then be just another special case of the Preparation Uncertainty Relation. The $\Delta P$ here would be the total variance of the momentum distribution after the measurement, i.e., not really that part of momentum uncertainty introduced by the measurement itself. It could be much smaller that the initial momentum spread. So this reduction of measurement uncertainty to preparation uncertainty is straightforward only if we know that the initial state has sharp momentum.

\subsection{Variance of covariant observables}

The curious constant .3047 in the relation we prove is perhaps not so strange if one notes that the same constant appears in the preparation uncertainty, if we choose to quantify the spread $\Delta Q$ of position not by the square root of a second moment, but by an absolute first moment. In fact, this is the way the constant was derived in Section 3.2 So it is suggestive to look for an interpretation of $\Delta Q$ and $\Delta P$ for measurement uncertainty, which would also bring the constant to $\hbar / 2$. (From talks I gave about the subject I know some colleagues find fault with any other constant). This can be done at the expense of the Kantorovich interpretation of the Monge distance 
as a worst case difference of expectation values, by using a cost function for transport, which grows quadratically with the distance (also known as the Wasserstein 2-metric).

This is especially suggestive for the purely covariant case, in which the marginals of joint measurements are equivalent to adding noise from an external source: one can then simply take the second moment of the noise. This approach has been suggested also by Holevo.

\subsection{Ozawa's Approach}

In a series of recent papers,Ozawa 17. M. Ozawa has also studied the measurement aspect of uncertainty. For this he considers measurements described as detailed couplings to an environment. Then one can explicitly point out a selfadjoint operator of the combined object-apparatus system which describes the momentum after the measurement. The 'perturbation of momentum' by the measurement is then represented by the difference of the momentum operators before and after the measurement interaction, and quantified by the expectation of the square of this operator.

This is definitely a departure from the operational approach to quantum mechanics, since this difference of non-commuting operators is not accessible in the given experiment. Of course, any operator represents an observable. But to find a device measuring just this operator is a highly non-trivial task. In contrast, in our approach only the statistics of measurements on the joint measuring device itself enters.

Nevertheless, there are interesting aspects in Ozawa's approach. In particular, his analysis applies to every input state separately, whereas our figures of merit involve a supremum over all input states. Further relationships remain to be clarified.

\section{References}

[1] W. Heisenberg, Über den anschaulichen Inhalt der quantentheoretischen Kinematik und Mechanik, (The perceptible content of the quantum theoretical kinematics and mechanics), Z.Phys 43 (1927) 172-198

[2] E.H. Kennard, Z.Phys. 44 (1927)326

[3] H.P. Robertson, Phys.Rev. 34 (1929)163

[4] K. Husimi, Proc. Phys. Math. Soc. Japan 22 (1940)264.

[5] A.S. Holevo, Rep. Math. Phys. 16 (1979) 385-400

[6] G. Monge, Mémoire sur la théorie des déblais et de remblais. Histoire de l'cadémie Royale des Sciences de Paris, avec les Mémoires de Mathématique et de Physique pour la même année, pages 666-704, 1781.

[7] L. Kantorovich, On the translocation of masses. C.R. (Doklady) Acad. Sci. URSS (N.S.) 37 (1942)199-201

[8] L.A. Caffarelli, M. Feldman, and R.J. McCann, J. Amer. Math. Soc. 15 (2002) $1-26$

[9] N. Bourbaki, Éléments de Mathématique, Intégration, (Chap. III), Hermann, Paris 1952

[10] J. Dieudonné, Foundations of Modern Analysis, (Chapter 13), Academic Press, 1970

[11] R.F. Werner, Found Phys 13 (1983) 859-881 
[12] J. von Neumann, Math. Ann. 104 (1931), 570

[13] A.S. Holevo, Probabilistic and statistical aspects of quantum theory, (NorthHolland, Amsterdam 1982)

[14] E.B. Davies, Quantum theory of open systems, (Academic Press, London 1976)

[15] R.F. Werner, J. Math. Phys. 25 (1984) 1404-1411

[16] E. Hewitt, K.A. Ross: Abstract harmonic analysis, Vol.I, Chapter IV. 17 (Springer, Berlin 1963)

[17] M. Ozawa, Phys. Rev. A 67 (2003), 042105, Phys. Lett. A 318 (2003) 21-29, Phys. Lett. A 320 (2004), 367-374, and quant-ph/0307057 